\title{
La métallurgie des poudres appliquée aux multimatériaux
}

\author{
Y. Le Guennec ${ }^{1}$, P. Dorémus ${ }^{1, a}$, L. Lazzarotto ${ }^{2}$, F. Dore ${ }^{2}$, Y. Kamdem ${ }^{3}$, D. Bouvard ${ }^{3}$, \\ A. Thomazic ${ }^{3}$, C. Pascal ${ }^{3}$, J.-M. Chaix ${ }^{3}$, J.M. Auger ${ }^{4}$, F. Valdivieso ${ }^{4}$ et P. Goeuriot ${ }^{4}$ \\ 1 INPG, Laboratoire 3S-R, Domaine Universitaire, 38041 Grenoble, France \\ ${ }^{2}$ CETIM, 7 rue de la presse, BP 802, 42952 Saint-Étienne Cedex 9, France \\ 3 Laboratoire SIMAP, INP Grenoble/CNRS/UJF, BP 75, 38402 Saint-Martin d'Hères Cedex, France \\ 4 ENSMSE-MPE, 154 cours Fauriel, 42023 Saint-Étienne Cedex 1, France
}

Reçu le 6 octobre 2008, accepté le 19 janvier 2009

\begin{abstract}
Résumé - Plusieurs laboratoires et centres techniques ont commencé à travailler sur la possibilité de réaliser des composés multimatériaux via la Métallurgie des Poudres. Dans un tel cas, ce procédé permet d'éviter le problème de l'assemblage et d'obtenir une production aux cotes. Les compressions dynamique $(\mathrm{CGV})$ et conventionnelle sont deux voies possibles qui pourront être comparées grâce à une presse de laboratoire instrumentée pour l'analyse des phénomènes. Pour certaines poudres, la CGV permet d'améliorer les propriétés à vert et de modifier favorablement les conditions de frittage. La compression est suivie d'un frittage conventionnel. Différents types de poudres (poudres métalliques, poudres dures mais également des poudres céramiques) sont utilisés dans ce projet permettant ainsi de proposer de nombreuses applications. Cette présentation montre les premiers résultats obtenus par le groupe de recherche.
\end{abstract}

Mots clés : Compression haute vitesse / mise en forme / frittage / multimatériaux / acier/carbure / composite à matrice céramique / essais / simulation numérique

\begin{abstract}
Powder metallurgy applied to multimaterials. Many laboratories and technical centres have begun to work on the possibility of creating multimaterial composites using Powder Metallurgy. This process can be used to avoid assembly problems and to obtain dimensionally accurate products. Dynamic compaction ('High-Speed Compaction') and conventional compaction are two possible methods, which can be compared using a laboratory press equipped with test instrumentation. For certain powders, High-Speed Compaction can improve the properties in the green state and apply favourable changes to the sintering conditions. Compaction is followed by conventional sintering. Various types of powder (metal powders, hard powders and ceramic powders) are used in this project, giving rise to many possible applications. This presentation shows the initial results obtained by the research group.
\end{abstract}

Key words: High-speed compression / formatting / sintering / multi-material / steel/carbide / ceramic matrix composites / testing / numerical simulation

\section{Introduction}

La métallurgie des poudres est un procédé permettant d'élaborer un matériau solide à partir de poudres métalliques sans passer par la fusion mais mettant en ouvre des techniques thermiques et/ou mécaniques. Cette méthode comprend, généralement, une phase de compression de la poudre puis de frittage pendant laquelle

\footnotetext{
a Auteur pour correspondance : pierre.doremus@hmg.inpg.fr
}

la pièce est chauffée afin d'obtenir ses caractéristiques mécaniques finales.

Plusieurs procédés d'élaboration sont utilisés aujourd'hui. Parmi eux, la compression grande vitesse (CGV) semble prometteuse. Cette technique émergente et préindustrielle est une forme de compression uniaxiale en matrice qui diffère du procédé classique par la façon dont la force de pressage est appliquée. Les études précédentes effectuées sur ce type de compression démontrent que, sur certaines poudres, de meilleures propriétés mécaniques sont obtenues $[1,2]$. 
La métallurgie des poudres permet également d'envisager la mise en forme de multimatériaux difficilement concevable par d'autres procédés. Cela ouvre de nouvelles perspectives de conception de produits, en combinant différentes fonctions (mécaniques, thermiques,...) au sein d'une même pièce. Cependant, la réalisation de tels produits n'est envisageable que lorsque les matériaux à mettre en œuvre peuvent être comprimés en respectant un contrôle géométrique des couches et surtout frittés suivant un même cycle thermique. Ainsi, la conception de multimatériaux pose des défis aussi bien au niveau de la compression que du frittage et la possibilité d'utiliser une compression dynamique pourrait permettre, grâce à de meilleures propriétés à vert, d'en résoudre une partie.

Dans l'optique de mettre en forme ces composés multimatériaux-multifonction, l'étude d'exemples simples de types bicouche ou bicylindre sera effectuée. Les différentes phases de conception seront simulées numériquement afin de mieux cerner les problématiques des différentes étapes. Plusieurs laboratoires (L3S-R, ENSMSE-MPE, SIMAP), le CETIM et des groupes industriels collaborent afin de résoudre les difficultés scientifiques et techniques rencontrées.

Cet article présente l'équipement à disposition, les premières simulations numériques de la compression dynamique, ainsi que l'avancement sur l'étude du frittage de multimatériaux sur différents types de poudres (un bimatériau acier/carbures cémentés et un composite à matrice céramique).

\section{La compression dynamique}

Deux types de presse dynamique sont utilisés, une presse de laboratoire située au laboratoire L3S-R et une presse hydraulique de formage grande vitesse faisant partie d'une plateforme technologique régionale implantée au CETIM de Saint-Etienne.

\subsection{Les presses}

\subsubsection{La presse de laboratoire}

Le dispositif, situé au laboratoire L3S-R (Grenoble, France), est une presse de laboratoire capable de développer une énergie de $380 \mathrm{~J}$. Un ressort est comprimé pour propulser une masse de $5,3 \mathrm{~kg}$ à des vitesses allant jusqu'à $12 \mathrm{~m} . \mathrm{s}^{-1}$. L'énergie cinétique de la masse est utilisée pour impacter un poinçon qui comprime la poudre contenue dans une matrice. L'énergie de l'impact est pilotée via un capteur d'effort qui mesure la compression du ressort et est vérifiée par un capteur de vitesse optique situé avant l'impact.

Les outils sont de diamètre $12,5 \mathrm{~mm}$ en acier CMD 8-5. Les poinçons supérieur et inférieur ainsi que la matrice sont instrumentés de jauges de déformations afin de connaître les contraintes axiales et radiales appliquées à la poudre.

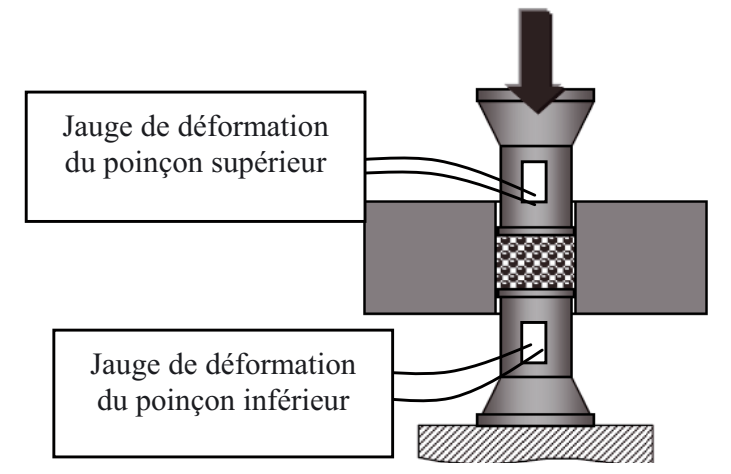

Fig. 1. Dispositif de compression.

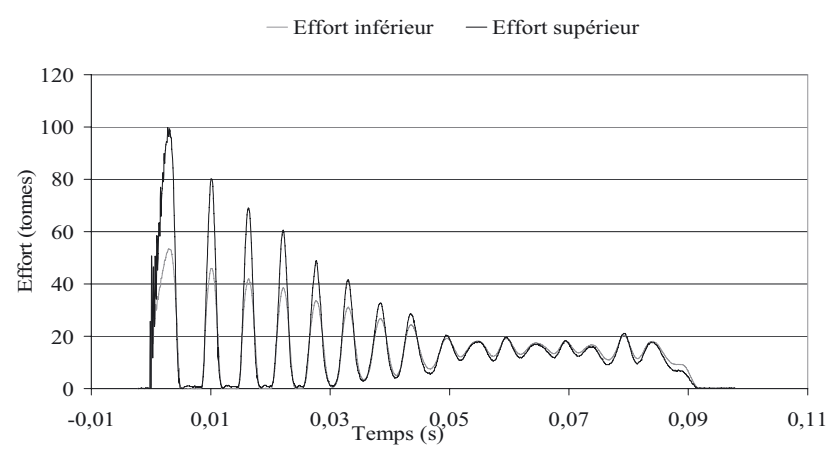

Fig. 2. Allure des pressions supérieure et inférieure donnée par les capteurs pendant la compression d'une poudre de fer avec la presse hydraulique du CETIM.

\subsubsection{La presse hydraulique}

Le CETIM de Saint-Etienne est équipé d'une presse dynamique hydraulique capable de développer une énergie de $20 \mathrm{~kJ}$ en imposant à son coulisseau une vitesse pouvant aller jusqu'à $11 \mathrm{~m} . \mathrm{s}^{-1}$. Une masse de $350 \mathrm{~kg}$ propulsée à grande vitesse sur une distance de $160 \mathrm{~mm}$ vient impacter le poinçon supérieur.

Cet équipement offre une fonctionnalité nouvelle dite «multi-coups » avec une fréquence de frappe de 5 coups par seconde. Cette fonctionnalité ou stratégie de compression, peut s'avérer extrêmement intéressante pour réaliser des pièces très massives $(3 \mathrm{~kg})$ avec une machine de taille modeste.

\subsection{Observations expérimentales et simulation numérique}

Après avoir rempli la matrice d'une poudre métallique et placé les outils, on vient impacter à grande vitesse le poinçon supérieur pour comprimer la poudre (Fig. 1).

\subsubsection{Signaux des capteurs}

La presse hydraulique montre une succession de chocs probablement due à des pics de pression d'huile dans la presse [2] (Fig. 2). La presse de laboratoire montre, quant 


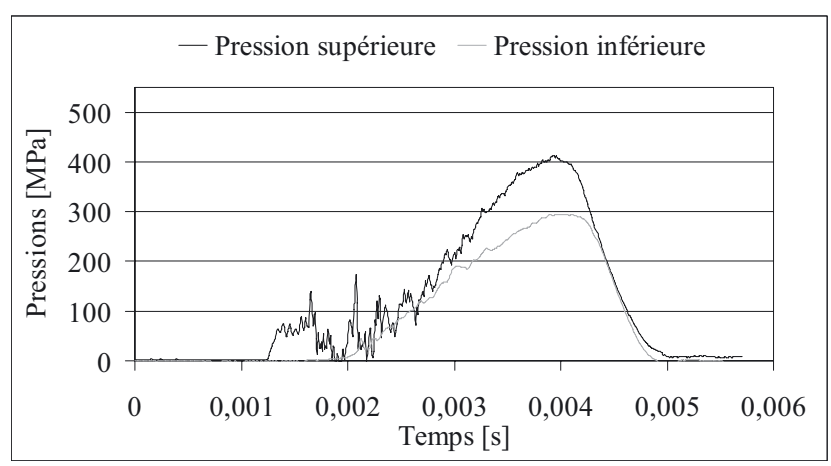

Fig. 3. Allure des pressions supérieure et inférieure $2 / 6$ donnée par les capteurs pendant la compression d'une poudre de fer avec la presse de laboratoire.

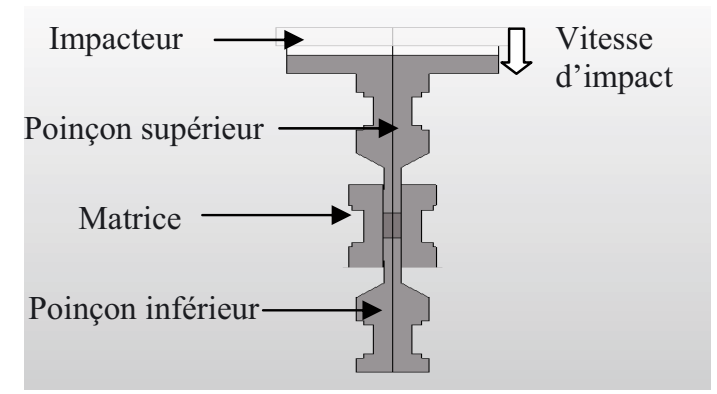

Fig. 4. Géométrie du dispositif de compression en simulation numérique.

à elle, un signal en plusieurs phases (Fig. 3) dû à la dynamique du choc avec, lors de l'impact, un signal correspondant à l'effort nécessaire pour vaincre l'inertie du poinçon supérieur. Néanmoins, le temps de compaction reste du même ordre de grandeur et nous sommes bien, dans les 2 cas, en compression grande vitesse.

\subsubsection{Simulation numérique}

On utilise le logiciel de calcul par éléments finis ABAQUS explicit pour simuler la compression dynamique de la presse de laboratoire. Bien que la loi de comportement utilisée pour la poudre soit celle utilisée en compression classique, on retrouve les ordres de grandeur des pressions et du temps de compaction (Figs. 4 et 5). Les avantages de la compression dynamique en vue de la conception de multimatériaux restent à définir et l'intérêt d'un modèle numérique permettant de prédire les comportements des poudres lors d'une telle compression est évident pour cette application.

Parallèlement, des études sont menées sur le frittage de deux types de multimatériaux : un matériau bicouche acier/carbures cémentés et un composite à matrice céramique.

\section{Le frittage}

La compression et le frittage de structures composées de différentes poudres peuvent s'avérer difficiles, quand les

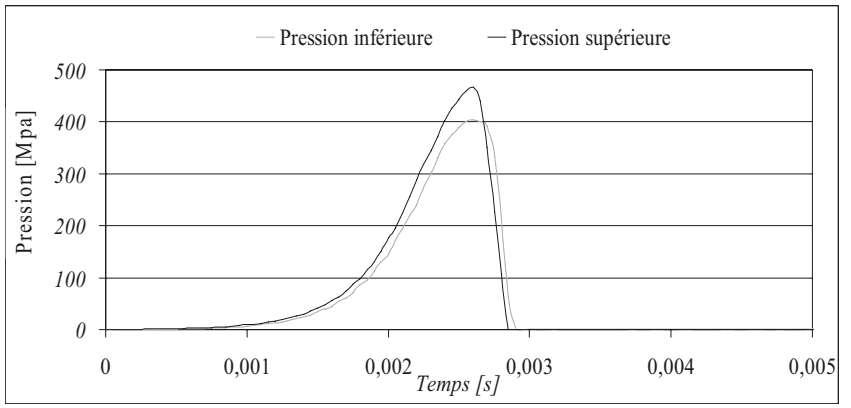

Fig. 5. Pressions supérieure et inférieure sur la surface de l'échantillon obtenues numériquement.

propriétés thermomécaniques et physiques des matériaux associés sont très différentes. Même dans les cas les moins critiques, se posent inévitablement des problèmes de densification hétérogène, de déformations différentielles, de contrôle d'interface et de contraintes internes, conduisant éventuellement à la création de défauts. Pour comprendre ces phénomènes et optimiser les paramètres d'élaboration, il est intéressant de pouvoir effectuer des simulations numériques de chaque phase du procédé. L'étude vise donc à développer des lois de comportement intégrées dans un code de calcul par éléments-finis pour simuler le frittage de structures comprenant différentes poudres métalliques ou céramiques.

Dans un premier temps, des données issues de la littérature sur des matériaux modèles sont utilisées. Parallèlement, de nouvelles données expérimentales sont acquises sur deux types de multimatériaux associant des propriétés de dureté et de résilience : un matériau bicouche acier/carbures cémentés et un composite à matrice céramique.

\subsection{Simulation du frittage d'un bicouche}

Le frittage d'une structure composée de matériaux présentant des cinétiques de frittage différentes peut induire des contraintes à l'intérieur de chaque matériau et aux interfaces, ainsi que des distorsions de la structure. La simulation numérique permet d'estimer ces effets et de proposer des solutions pour les réduire, en jouant par exemple sur le cycle de frittage ou la géométrie. À titre d'illustration nous présentons la simulation du frittage d'un cylindre bicouche ( $\varnothing 10 \mathrm{~mm}$, hauteur $5 \mathrm{~mm}$ ) alumineWC-Co, en supposant une interface collée entre les deux matériaux. Il s'agit d'un exemple théorique, non réalisable expérimentalement, choisi car nous disposions de lois de comportement pour ces deux poudres $[3,5,6]$. Le calcul est réalisé avec le logiciel ABAQUS, les lois de comportement étant introduites à l'aide de la subroutine CREEP. Nous supposons une densité initiale décroissante dans la hauteur du bicouche, avec une moyenne de $52 \%$ de densité relative dans la partie haute en $\mathrm{WC}-\mathrm{Co}$ et de $57 \%$ dans la partie basse en alumine. La pièce est soumise à un cycle classique de frittage de $\mathrm{WC}-\mathrm{Co}$ avec une évolution de la température supposée homogène allant jusqu'à $1410{ }^{\circ} \mathrm{C}$. 


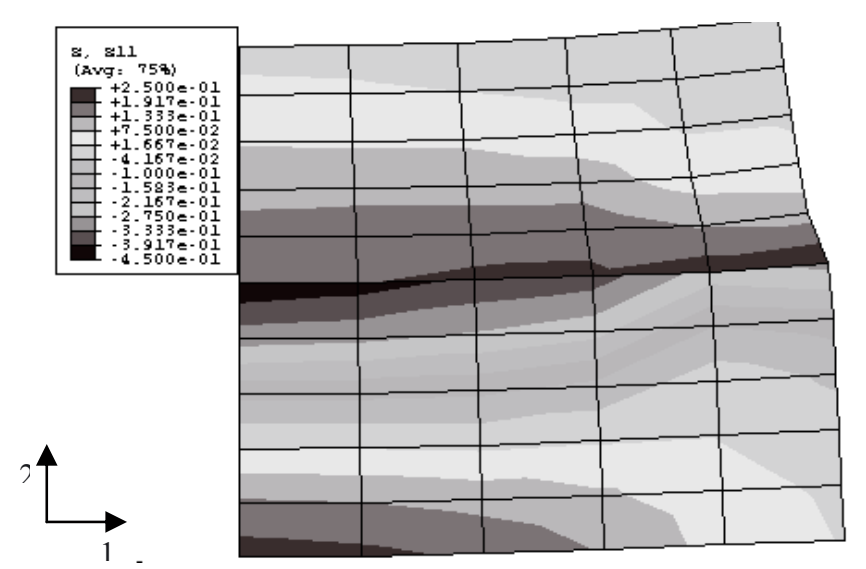

Fig. 6. Distribution des contraintes normales horizontales en $\mathrm{MPa}$ dans le bicouche $\mathrm{WC}-\mathrm{Co} /$ alumine en cours de frittage à $3 / 61250{ }^{\circ} \mathrm{C}$.

À l'issue de ce cycle WC-Co est quasiment dense alors que l'alumine atteint une densité relative de $72 \%$.

Les résultats de simulation montrent que des contraintes normales apparaissent au voisinage de l'interface en cours de frittage. Entre $1100{ }^{\circ} \mathrm{C}$ et $1350{ }^{\circ} \mathrm{C}$, $\mathrm{WC}-\mathrm{Co}$ densifie fortement alors que l'alumine est quasiment à densité constante, ce qui entraîne des contraintes significatives, jusqu'à $0,5 \mathrm{MPa}$, de traction dans $\mathrm{WC}-\mathrm{Co}$ (Fig. 6) et de compression dans l'alumine. Ce phénomène s'inverse à plus haute température quand la densification de l'alumine commence. On observe aussi la flexion de la pièce due à la différence de retrait.

\subsection{Frittage de multimatériaux}

\subsubsection{Bi-matériaux acier/carbures de tungstène}

Les bi-matériaux acier/carbures de tungstène permettent d'associer des propriétés de résilience et de dureté [4]. Les mélanges de poudres base $\mathrm{Fe}(-\mathrm{WC}-\mathrm{C})$ et base WC $(-\mathrm{Fe}-\mathrm{C})$ sont préparés par attrition, puis comprimés uni-axialement en matrice $(P=600 \mathrm{MPa})$. Les densités relatives à vert sont de $74 \%$ et $69 \%$ pour les comprimés base Fe et base WC respectivement.

Le traitement thermique se déroule en deux étapes (Fig. 7a) : le premier palier permet l'élimination du liant organique (étape de déliantage) nécessaire à la compression et à la tenue à vert des comprimés. Une étude thermogravimétrique conduite sur chaque matériau montre que le déliantage est complet pour des températures de $340^{\circ} \mathrm{C}$ et $360^{\circ} \mathrm{C}$ pour les comprimés base Fe et base $\mathrm{WC}$ respectivement. Le deuxième palier à $1300^{\circ} \mathrm{C}$ constitue l'étape de frittage. Le retrait observé pour chaque matériau est isotrope et de l'ordre de $8 \%$ pour le comprimé base Fe et de $10 \%$ pour le comprimé base WC. Les densités après frittage sont voisines de $93 \%$.

La figure 7 a représente les courbes de retrait axial et le cycle thermique en fonction du temps pour les matériaux base Fe et base WC. Les courbes se différencient par l'amplitude des variations dimensionnelles, les vitesses

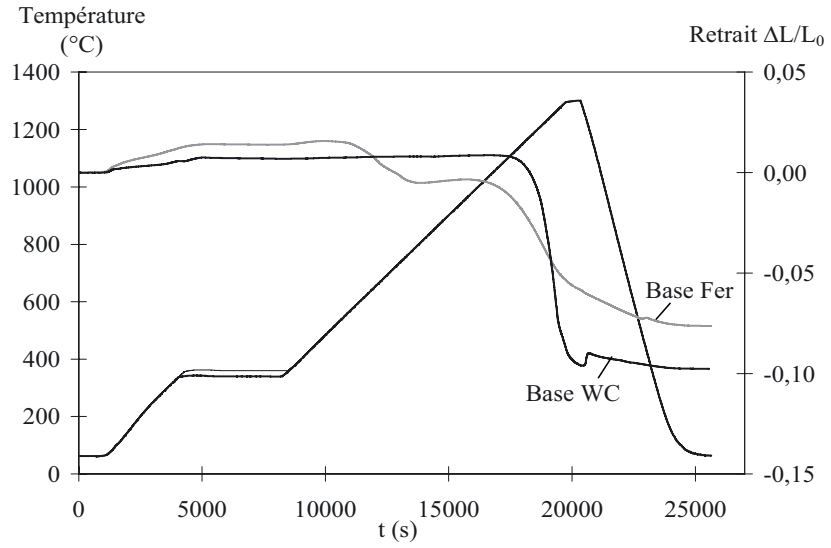

(a)

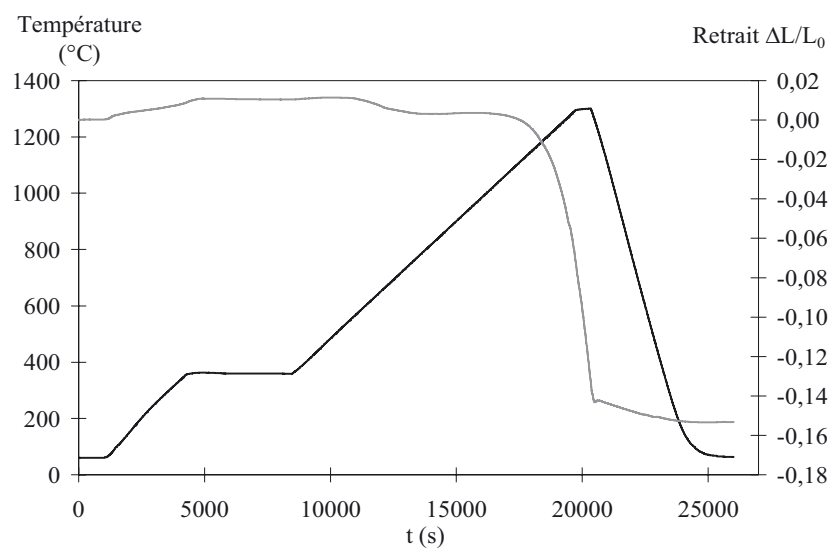

(b)

Fig. 7. Cycle de déliantage-frittage et courbes de retrait en fonction du temps (a) pour les mono-matériaux base Fe et base WC (b) pour le bi-matériau base Fe/base WC.

de retrait et les températures caractéristiques. En particulier, le retrait atteint une vitesse maximale pour des températures de l'ordre de $1220{ }^{\circ} \mathrm{C}$ pour le matériau base Fe et de $1265^{\circ} \mathrm{C}$ pour le matériau base WC.

Ces comportements différents des deux « monomatériaux » lors du frittage constituent un obstacle à l'obtention d'une bonne cohésion lors de l'élaboration de bi-matériaux. Cependant, les premiers essais de frittage de bi-matériaux (constitués de $50 \%$ en volume de chaque mélange) présentent des variations dimensionnelles différentes de celles de ses matériaux constitutifs pris isolément (Fig. 7b). Le retrait de chaque couche n'est alors plus isotrope : le retrait radial est inférieur au retrait axial. Cette différence pourrait s'expliquer par un transfert de matière entre les deux couches constitutives du bi-matériau.

Le cycle thermique de frittage devra être optimisé pour limiter les phénomènes d'endommagement à l'interface, qui peuvent résulter des contraintes internes créées par les différences de retrait, et pour maintenir l'intégrité chimique du bi-matériau, en particulier en maîtrisant les phénomènes de réactivité à l'interface et de transfert de matière entre les couches. 


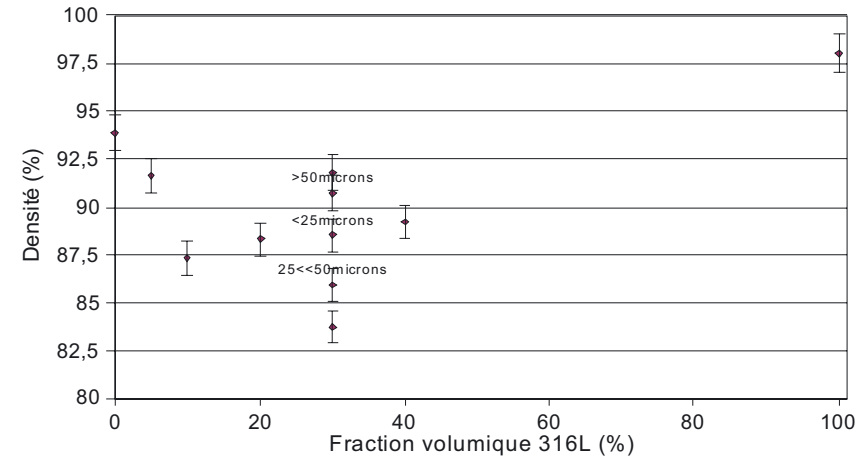

Fig. 8. Évolution du taux de densification des composites après frittage.

\subsection{2 Étude du composite à matrice céramique}

L'idée maîtresse de cette étude est de réaliser un stratifié composé d'une alternance de couches ayant des propriétés de ductilité et de résilience mais dont on augmenterait la dureté par dispersion de céramique, et de couches extrêmement dures mais non fragiles constituées d'un composite à matrice céramique contenant une dispersion de phase métallique. La même phase métallique assurera la continuité des propriétés de résilience et de ductilité pour l'ensemble de la pièce.

Le choix des matériaux et leurs proportions relatives dans chacune des couches et l'architecture des couches (épaisseur relatives, sens de l'alternance) seront les paramètres à optimiser afin d'assurer la faisabilité d'un tel sandwich dans un premier temps (retrait compatible en cofrittage, coefficient de dilatation adapté, qualité des interfaces), puis pour régler les propriétés finales da la pièce. Le choix des matériaux s'est porté sur l'acier 316L pour le métal et l'alumine SM8 pour la céramique. Ces poudres sont formulées par les industriels pour s'assurer de leur compressibilité. Des mélanges homogènes, de 0 à 40 vol. \% de métal, sont réalisés par agitation dans un mélangeur à billes. Le pressage des poudres est réalisé en matrice. Le frittage est réalisé selon un cycle de température permettant un déliantage non dégradant pour la pièce. Le choix de la température de frittage $\left(1410^{\circ} \mathrm{C}\right)$ est guidé par la température d'apparition d'un liquide métallique vers $1435{ }^{\circ} \mathrm{C}$ et $\mathrm{du}$ fait d'un étroit domaine de température de fusion totale. Ces premiers résultats montrent que la phase métallique nuit au frittage de l'alumine et que cette dégradation dépend de la classification granulométrique du métal (Fig. 8).

L'observation macrostructurale en microscopie optique de la pièce (30 vol. \% 316L) confirme l'homogénéité de la dispersion métallique, tandis qu'un plus fort grossissement (Fig. 9) précise que la porosité résiduelle est essentiellement présente dans la phase céramique, les amas métalliques étant quasi denses. On remarque par MEB, que l'interface céramique-métal ne présente pas de décohésion en dépit des coefficients de dilatation très différents des deux phases. Enfin l'analyse par DRX montre la présence d'une phase spinelle de type (Fe, Cr) $\mathrm{Al}_{2} \mathrm{O}_{4}$ dont on ne peut révéler l'emplacement

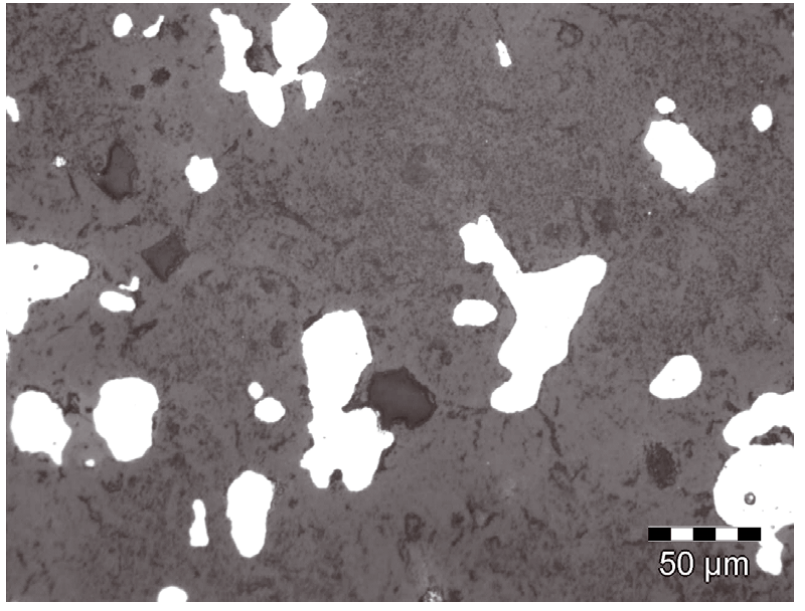

Fig. 9. Microstructure des composites.

par visualisation de faces polies. L'analyse des profils du fer et de l'aluminium autour d'une interface montre clairement une diffusion plus importante du fer dans le grain de céramique que le $\mathrm{Al}$ dans le grain métallique.

\section{Conclusion}

L'étude de la compression grande vitesse a débuté. Les avantages possibles en vue de la mise en forme de multimatériaux sont encore à étudier et la modélisation numérique du procédé sera une aide précieuse dans l'étude d'une telle compression. Les premiers essais de frittage de multimatériaux ont mis en évidence les difficultés que l'on est amené à rencontrer. Ainsi, les phénomènes observés à l'interface (réactivité, transfert de matière) et les problèmes liés aux différences de retrait devront être maîtrisés. La possibilité de simuler numériquement le frittage pourrait permettre de répondre à quelques unes de ces questions.

Remerciements. La Direction Générale des Entreprises, Le conseil Général de la Loire ainsi que Saint-Étienne Métropole pour leur soutien financier au projet «MULTIMAT », dans le cadre du Pôle de Compétitivité ViaMéca.

La Fondation CETIM pour son soutien financier dans le cadre du projet « Produits Multi-matériaux et Multifonctions $»$.

Note de l'éditeur : les épreuves de l'article n'ont pas été corrigées par les auteurs.

\section{Références}

[1] P. Doremus, F. Duwa, P. Francois, G. Puente, C. Allibert, High velocity compaction, In World congress on powder metallurgy \& particulate materials, Vol. 4, Metal Powder Industries Federation, MPIF, Orlando, Florida, USA, 2002, pp. 96-110 
[2] D. Souriou, P. Goeuriot, O. Bonnefoy, G. Thomas, S. Drapier, S. Bourdin, L. Lazzarotto, Comparison of conventional and high velocity compaction of alumina powder, CIMTEC 2006, Acireale (Sicile), Adv. Sci. Tech. 45-53 (2006) 893-898

[3] O. Alvain, D. Bouvard, P. Doremus, Proc. 2000 powder metallurgy world congress, Kyoto, Japan society of powder and powder metallurgy, 2000, pp. 613-616,

[4] C. Pascal, F. Doré, D.R. Amador, P. Doremus, J.M. Chaix, C.H. Allibert, Design of multimaterial processed by powder metallurgy for multifunctional application, Proceedings of the 4th international conference on science, Technology and applications of sintering, Grenoble, France, 29 août-1 septembre 2005, pp. $212-215$

[5] O. Gillia, D. Bouvard, Mater. Sci. Eng. A 279 (2000) 185-191

[6] H.G. Kim, O. Gillia, D. Bouvard, J. Eur. Ceram. Soc. 23 (2003) 1675-1685 